\title{
Nanostructure and velocity of field-driven solid-on-solid interfaces moving under a phonon-assisted dynamic
}

\author{
G. M. Buendía, ${ }^{1, *}$ P. A. Rikvold, $, 2,3,4, \dagger$ M. Kolesik, ${ }^{5,6, \$}$ K. Park, ${ }^{7,8}$ and M. A. Novotny ${ }^{8, \|}$ \\ ${ }^{1}$ Department of Physics, Universidad Simón Bolivar, Caracas 1080, Venezuela \\ ${ }^{2}$ School of Computational Science, Florida State University, Tallahassee, Florida 32306-4120, USA \\ ${ }^{3}$ Center for Materials Research and Technology and Department of Physics, \\ Florida State University, Tallahassee, Florida 32306-4350, USA \\ ${ }^{4}$ National High Magnetic Field Laboratory, Tallahassee, Florida 32310, USA \\ ${ }^{5}$ Institute of Physics, Slovak Academy of Sciences, Bratislava, Slovak Republic \\ ${ }^{6}$ College of Optical Sciences, University of Arizona, Tucson, Arizona 85721, USA \\ ${ }^{7}$ Department of Physics, Virginia Polytechnic Institute and State University, Blacksburg, Virginia 24061, USA \\ ${ }^{8}$ Department of Physics and Astronomy and $\mathrm{HPC}^{2}$ Center for Computational Sciences, \\ Mississippi State University, Mississippi 39762-5167, USA
}

(Received 3 May 2007; revised manuscript received 15 June 2007; published 23 July 2007)

\begin{abstract}
The nanoscopic structure and the stationary propagation velocity of $(1+1)$-dimensional solid-on-solid interfaces in an Ising lattice-gas model, which are driven far from equilibrium by an applied force, such as a magnetic field or a difference in (electro)chemical potential, are studied by an analytic nonlinear-response approximation [P. A. Rikvold and M. Kolesik, J. Stat. Phys. 100, 377 (2000)] together with kinetic Monte Carlo simulations. Here, we consider the case that the system is coupled to a two-dimensional phonon bath. In the resulting dynamic [K. Saito et al., Phys. Rev. E 61, 2397 (2000); K. Park and M. A. Novotny, Comput. Phys. Commun. 147, 737 (2002)], transitions that conserve the system energy are forbidden, and the effects of the applied force and the interaction energies do not factorize (a so-called hard dynamic). In full agreement with previous general theoretical results, we find that the local interface width changes dramatically with the applied force. However, in contrast with other hard dynamics, this change is nonmonotonic in the driving force. Results are also obtained for the force dependence and anisotropy of the interface velocity, which also show differences in good agreement with the theoretical expectations for the differences between soft and hard dynamics. However, significant differences between theory and simulation are found near two special values of the driving force, where certain transitions allowed by the solid-on-solid model become forbidden by the phonon-assisted dynamic. Our results show that different stochastic interface dynamics that all obey detailed balance and the same conservation laws nevertheless can lead to radically different interface responses to an applied force. Thus, they represent a significant step toward providing a solid physical foundation for kinetic Monte Carlo simulations.
\end{abstract}

DOI: 10.1103/PhysRevB.76.045422

PACS number(s): 68.37.- d, 68.35.Ct, 68.65.-k, 75.60.Jk

\section{INTRODUCTION}

Moving internal boundaries or interfaces separating different regions are present in many problems in nature, and the challenge of understanding the dynamics of such processes has become increasingly important. In recent years, considerable efforts have been made toward understanding the large-scale structures of growing interfaces. ${ }^{1,2}$ In contrast, there has been little work related to the microscopic and nanoscopic scales. This is surprising since the nanoscopic interface structure plays a crucial role in important interface properties such as mobility and catalytic and chemical activity. Technologically, as the sizes of the smallest man-made structures decrease, interfacial properties become essential and even dominant. Nanoscale assemblies with highly ordered building blocks, such as quantum dots ${ }^{3,4}$ and quantum wires, must be fabricated on a surface or through an interface.

The basic mechanisms of interface growth are complex and often unknown. A standard way to deal with this problem is constructing a stochastic model that reproduces essential features. However, extreme care has to be taken with this approach. Recent studies indicate that different stochastic dynamics, even when they have the same conserved quantities and satisfy detailed balance, lead to important differences in the nanostructure of field-driven interfaces. ${ }^{5-9}$ Surfaces driven by hard dynamics (in which the single-site transition rates cannot be factorized into one term that depends only on the interaction energies and a second term that depends only on the field energies, in contrast with soft dynamics for which this factorization is possible ${ }^{10}$ ), such as Glauber, Metropolis, and the two-step transition dynamics approximation (TDA), ${ }^{11,12}$ have a strong dependence on the applied field. For all hard dynamics studied so far, the average step height increases dramatically with increasing field. In contrast, interfaces driven by soft dynamics, such as the soft Glauber ${ }^{6}$ and the one-step dynamics (OSD), $, 13,14$ are at most only weakly dependent on the field and relatively smooth. Furthermore, interfaces driven by hard dynamics, such as Glauber $^{7}$ and TDA, ${ }^{9}$ display significant asymmetry between the spin populations on their leading and trailing edges, while interfaces moving under soft dynamics either display no (soft Glauber ${ }^{6}$ ) or only weak $\left(\mathrm{OSD}^{9}\right)$ anisotropy. 
In this paper, we study by kinetic Monte Carlo (MC) simulation and a dynamic mean-field approximation the motion of a Burton-Cabrera-Frank solid-on-solid (SOS) interface ${ }^{15}$ that evolves under a nonconservative dynamic resulting from coupling the system to a phonon heat bath. SOS interfaces belong to the Kardar-Parisi-Zhang (KPZ) dynamic universality class, ${ }^{1,16}$ in which the macroscopic, stationary distribution for moving interfaces is Gaussian, corresponding to a random walk with independent increments. The phononassisted dynamic is obtained by introducing a weak, linear coupling between a square-lattice Ising quantum ferromagnet and a phonon (i.e., bosonic) heat bath attached to the spin system. The transition rates have been calculated using the quantum-mechanical density matrix equation ${ }^{17-21}$ and most recently also by the lattice-frame method. ${ }^{22}$ Both methods give consistent results. The resulting dynamic is quite different from the Glauber dynamic, which can be similarly derived from coupling to fermionic baths. ${ }^{23}$ In particular, for phonon baths of dimension greater than 1 , the phononassisted dynamic prohibits transitions that conserve the system energy, even if they are allowed by the SOS restriction. As a result, the model becomes nonergodic near special values of the driving field, and the interfaces can get stuck in long-lived, metastable, or permanently "frozen" states. The average step height and propagation velocity therefore become nonmonotonic functions of the field. Phonon-assisted dynamics are relevant in a great variety of physical phenomena, ranging from the nonlinear optical response of semiconductors ${ }^{24}$ to the dynamics of quantum dots. ${ }^{3,4}$ The derivation of a phonon-assisted stochastic dynamic is a significant step toward putting kinetic MC on a solid physical foundation.

In this paper, we derive analytic, approximate expressions for the interface propagation velocity as a function of field, temperature, and interface orientation. Our approach is based on a mean-field approximation that assumes that individual steps on the interface are statistically independent, ${ }^{6-8}$ i.e., short-range correlations are neglected. This limitation becomes apparent when we compare the analytical and simulated results.

The remainder of this paper is organized as follows. In Sec. II, we introduce the SOS interface model and give the transition rates for the phonon-assisted dynamic. Also in this section, we summarize the mean-field approximation for the time evolution of the single-step probability density function (pdf), as well as its stationary form. We further give expressions for the spin-class populations and interface velocity in terms of the applied field, the temperature, and the angle of the interface relative to the lattice axes. In Sec. III, we compare simulations and analytical predictions for the detailed stationary interfacial nanostructure, including the asymmetry of the simulated nonequilibrium interfaces. Summary and conclusions are provided in Sec. IV.

\section{MODEL AND DYNAMICS}

The SOS interfaces are described by the nearest-neighbor $S=1 / 2$ Ising Hamiltonian with anisotropic, ferromagnetic interactions $J_{x}$ and $J_{y}$ in the $x$ and $y$ directions, respectively,

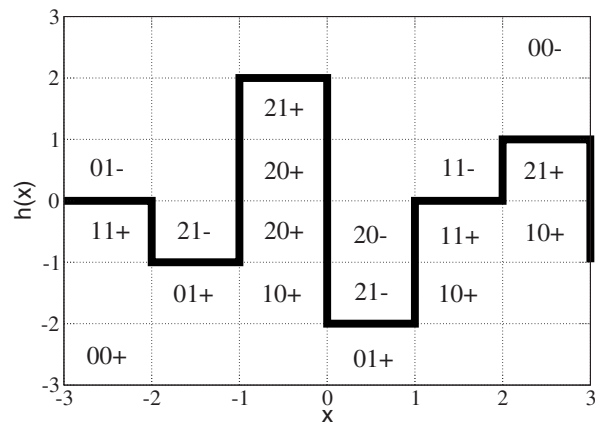

FIG. 1. A short segment of an SOS interface $y=h(x)$ between a positively magnetized phase (or "solid" phase in the lattice-gas picture) below and a negative (or "fluid") phase above. The step heights are $\delta(x)=h(x+1 / 2)-h(x-1 / 2)$. Interface sites representative of the different SOS spin classes (see Table I) are marked with the notation $j k s$, explained in the text. Sites in the uniform bulk phases are $00-$ and $00+$. This interface was generated with a symmetric step-height distribution, corresponding to $\phi=0$.

$$
\mathcal{H}=-\sum_{x, y} s_{x, y}\left(J_{x} s_{x+1, y}+J_{y} s_{x, y+1}+H\right)
$$

where $s_{x, y}= \pm 1, \Sigma_{x, y}$ runs over all sites, and the applied field $H$ is the driving force. The interface is introduced by fixing $s_{x, y}=+1$ and -1 for large negative and positive $y$, respectively. Without loss of generality, we take $H \geqslant 0$, such that the interface on average moves in the positive $y$ direction. This Ising model is equivalent to a lattice-gas model with local occupation variables $c_{x, y} \in\{0,1\} .{ }^{25,26}$ Specifically, we identify $s=+1$ with $c=1$ (occupied or "solid") and $s=-1$ with $c=0$ (empty or "fluid").

The SOS model considers an interface in a lattice gas or $S=1 / 2$ Ising system on a square lattice of unit lattice constant as a single-valued integer function $h(x)$ of the $x$ coordinate, with steps $\delta(x)=h(x+1 / 2)-h(x-1 / 2)$ at integer values of $x$. A typical SOS interface configuration is shown in Fig. 1. In this paper, the two possible states of the site $(x, y)$ are denoted by the two Ising spin values $s_{x, y}= \pm 1$. (In order that the step positions and the interface heights be integer as stated above, we place the spins at half-integer values of $x$ and $y$, i.e., at the centers of the unit cells separated by dotted lines in Fig. 1.)

The interface is made to evolve under the phonon-assisted dynamic, a single-spin-flip (nonconservative) set of transition rates that satisfies detailed balance for the allowed transitions. In most cases, this ensures the approach to equilibrium, which in this case is a uniformly positive phase with the interface pushed off to positive infinity (for exceptions, see below). The dynamic is defined by the single-spin transition rates, $W\left(s_{x, y} \rightarrow-s_{x, y}\right)=W(\beta \Delta E)$. Here, $\beta$ is the inverse of the temperature $T$ (Boltzmann's constant is taken as unity), and $\Delta E$ is the energy change corresponding to a successful spin flip. The detailed-balance condition (valid for transitions between allowed states) is expressed as $W(\beta \Delta E) / W(-\beta \Delta E)=e^{-\beta \Delta E}$.

The transition rates for the phonon-assisted dynamic are defined as ${ }^{18,19}$ 

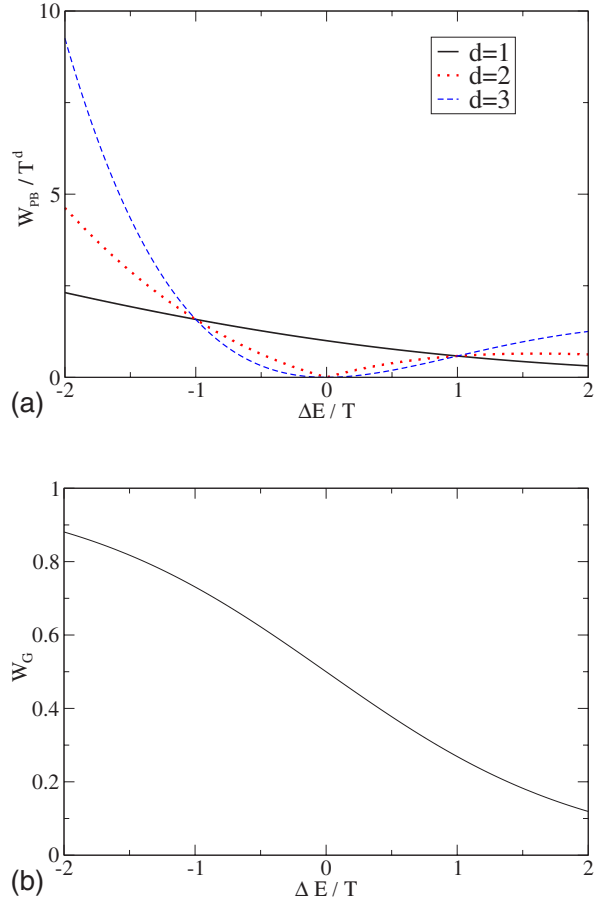

FIG. 2. (Color online) (a) The transition rates for the $d$-dimensional phonon-assisted dynamic, $W_{\mathrm{PB}}$, shown scaled by $T^{d}$ vs the energy difference $\Delta E$ scaled by $T$. (b) The transition rates for the standard Glauber dynamic, $W_{G}$, shown vs $\Delta E / T$.

$$
W_{\mathrm{PB}}(T, \Delta E)=\left|\frac{\Delta E^{d}}{e^{\beta \Delta E}-1}\right|,
$$

where $d \in\{1,2,3\}$ is the dimension of the bosonic heat bath. Physically, this rate is the product of three factors: the phonon occupation number $\left(e^{\beta|\Delta E|}-1\right)^{-1}$, the phonon density of states, proportional to $|\Delta E|^{d-1}$, and the magnetoelastic spinphonon coupling, proportional to $\left.|\Delta E|\right|^{18,22}$ For $d=2$ and 3, $W_{\mathrm{PB}}(T, \Delta E=0)=0$, while for $d=1$, the transition rate is nonzero and smooth at $\Delta E=0$. These transition rates are plotted in Fig. 2(a). For comparison, we also plot the transition rates for the Glauber dynamic in Fig. 2(b). In the present work, with the exception of Fig. 7, we use $d=2$, i.e, a twodimensional heat bath. Thus, the transition rates vanish linearly with $|\Delta E|$ near $|\Delta E|=0$. It should, however, be emphasized that the derivations of Eq. (2) are based on a weak, linear coupling of the phonon bath to the spin system. It is therefore possible that nonlinear and/or multiphonon effects may set a lower bound on physical transition rates for $\Delta E$ near zero and thus restore the ergodicity of the spin model. However, we note that recent experiments on phononmediated spin relaxation in a quantum dot shows a significant decrease in the relaxation rate for transitions involving $\Delta E$ near zero. ${ }^{4}$

Notice that the phonon-assisted transition rates cannot be factorized into one part that depends only on the interaction energy and another that depends only on the applied field; thus, it belongs to the class of dynamics defined as hard. ${ }^{6,10}$ In order to preserve the SOS configuration at all times, flips are allowed only at sites which have exactly one broken bond in the $y$ direction.
TABLE I. The spin classes in the anisotropic square-lattice SOS model. The first column contains the class labels, $j k s$. There are two other classes, $10 s$ and $20 s$, that also have nonzero populations in the SOS model but are not included because flipping a spin in any of them would produce an overhang or a bubble and is therefore forbidden. The second column contains the change in the total system energy resulting from reversal of a spin from $s$ to $-s, \Delta E(j k s)$. The third column contains the mean spin-class populations for general tilt angle $\phi$, with $\cosh \gamma(\phi)$ from Eq. (6).

\begin{tabular}{lcc}
\hline \hline Class, $j k s$ & $\Delta E(j k s)$ & $\langle n(j k s)\rangle$ \\
$01 s$ & $2 s H+4 J_{x}$ & $\frac{1-2 X \cosh \gamma(\phi)+X^{2}}{\left(1-X^{2}\right)^{2}}$ \\
$11 s$ & $2 s H$ & $\frac{2 X\left[\left(1+X^{2}\right) \cosh \gamma(\phi)-2 X\right]}{\left(1-X^{2}\right)^{2}}$ \\
$21 s$ & $2 s H-4 J_{x}$ & $\frac{X^{2}\left[1-2 X \cosh \gamma(\phi)+X^{2}\right]}{\left(1-X^{2}\right)^{2}}$ \\
\hline \hline
\end{tabular}

With the Ising Hamiltonian, there are only a finite number of different values of $\Delta E$. The spins can therefore be divided into ten classes, ${ }^{27-30}$ labeled by the spin value $s$ and the number of broken bonds between the spin and its nearest neighbors in the $x$ and $y$ directions, $j$ and $k$, respectively. The spin classes consistent with the SOS model are denoted $j k s$ with $j \in\{0,1,2\}$ and $k \in\{0,1\}$. They are shown in Fig. 1 and listed in Table I. At $H=0, \Delta E=0$ for transitions between 11 - and 11+ (diffusion of steps of unit height). Thus, these transitions are forbidden for $d=2$ and 3 . At $H=2 J_{x}$, the transitions forbidden for $d=2$ and 3 are between $01-$ and $21+$ [nucleation or elimination of a knob of stable $(+)$ phase on a smooth, horizontal interface]. For other values of (nonnegative) $H$, no transitions allowed by the SOS condition are forbidden.

In the SOS model and our analytical approximation, the heights of the individual steps are assumed to be statistically independent and identically distributed. This assumption is exact for $H=0 .{ }^{15}$ The step-height pdf is given by the interaction energy corresponding to the $|\delta(x)|$ broken $J_{x}$ bonds between spins in the columns centered at $(x-1 / 2)$ and $(x$ $+1 / 2)$ as

$$
p[\delta(x)]=Z(\phi)^{-1} X^{|\delta(x)|} e^{\gamma(\phi) \delta(x)} .
$$

The factor $X$ determines the width of the pdf, and $\gamma(\phi)$ is a Lagrange multiplier which maintains the mean step height at an $x$-independent value, $\langle\delta(x)\rangle=\tan \phi$, where $\phi$ is the overall angle between the interface and the $x$ axis. $Z(\phi)$ is a partition function that will be discussed below. In equilibrium, $X$ is simply the Boltzmann factor, $e^{-2 \beta J_{x}}$, which is independent of $H$. In previous papers, ${ }^{5,7}$ an expression for a field-dependent $X(T, H)$ was obtained, based on a dynamic mean-field approximation for the equation of motion for the single-step pdf together with a detailed-balance argument for the stationary state. This improved nonlinear-response approximation gives (see Ref. 7 for details of the calculation) 


$$
X(T, H)=e^{-2 \beta J_{x}}\left\{\frac{e^{-2 \beta H} W\left[\beta\left(-2 H-4 J_{x}\right)\right]+e^{2 \beta H} W\left[\beta\left(2 H-4 J_{x}\right)\right]}{W\left[\beta\left(-2 H-4 J_{x}\right)\right]+W\left[\beta\left(2 H-4 J_{x}\right)\right]}\right\}^{1 / 2},
$$

which is independent of $\gamma(\phi)$. The dependence on the specific dynamic is evidenced here by the presence of the transition rates associated with the reversal of a single spin, $W(\beta \Delta E)$. For $H=0, X$ is reduced to its equilibrium value, $X(T, 0)=e^{-2 \beta J_{x}}$. For soft dynamics, where the field and the interaction terms factorize, the $H$ dependence in Eq. (4) cancels out, while for hard dynamics, $X$ has a nontrivial dependence on $H$. Early results indicated that the SOS interfaces generated with the soft Glauber dynamic are indeed independent of $H^{6}{ }^{6}$ However, interfaces generated with the OSD dynamic, which is also soft, show a weak dependence on the interface structure of the field. ${ }^{9}$

The partition function for the interface is

$$
Z(\phi)=\sum_{\delta=-\infty}^{+\infty} X^{|\delta|} e^{\gamma(\phi) \delta}=\frac{1-X^{2}}{1-2 X \cosh \gamma(\phi)+X^{2}},
$$

where $\gamma(\phi)$ is given by

$$
e^{\gamma(\phi)}=\frac{\left(1+X^{2}\right) \tan \phi+\left[\left(1-X^{2}\right)^{2} \tan ^{2} \phi+4 X^{2}\right]^{1 / 2}}{2 X(1+\tan \phi)}
$$

(see details in Refs. 5 and 7). The mean spin-class populations, $\langle n(j k s)\rangle$, are all obtained from the product of the independent pdfs for $\delta(x)$ and $\delta(x+1)$. Symmetry of $p[\delta(x)]$ under the transformation $(x, \phi, \delta) \rightarrow(-x,-\phi,-\delta)$ ensures that $\langle n(j k-)\rangle=\langle n(j k+)\rangle$ for all $j$ and $k$. Numerical results illustrating the breakdown of this up-down symmetry for large $H$ are discussed in Sec. III. The general expressions for the class populations are given in the third column of Table I; details of the calculation can be found in Ref. 7 .

Whenever a spin flips from -1 to +1 , the corresponding column of the interface advances by one lattice constant in the $y$ direction. Conversely, the column recedes by one lattice constant when a spin flips from +1 to -1 . The corresponding energy changes are given in the second column in Table I. Since the spin-class populations on both sides of the interface are equal in this approximation, the contribution to the mean velocity in the $y$ direction from sites in the classes $j k-$ and $j k+$ becomes

$$
\left\langle v_{y}(j k)\right\rangle=W[\beta \Delta E(j k-)]-W[\beta \Delta E(j k+)] .
$$

The mean propagation velocity perpendicular to the interface becomes

$$
\left\langle v_{\perp}(T, H, \phi)\right\rangle=\cos \phi \sum_{j, k}\langle n(j k s)\rangle\left\langle v_{y}(j k)\right\rangle,
$$

where the sum runs over the classes included in Table I. It was shown in Ref. 7 that Eq. (8) reduces to the results for the single-step ${ }^{27,31-33}$ and the polynuclear growth ${ }^{31,34,35}$ models at low temperatures for large and small $\phi$, respectively. The spin-class populations listed in Table I can be calculated ex- plicitly by replacing $X$ with its corresponding value from Eq. (4).

In the next section, we show that the nonlinear-response approximation gives good agreement with MC simulations of driven SOS interfaces evolving under the phonon-assisted dynamic for a wide range of fields and temperatures. The main deviations between theory and simulations are seen for $H / J_{x}=0$ and 2, where some transitions allowed by the SOS restrictions have $\Delta E=0$ and thus are forbidden by the twodimensional phonon-assisted dynamic, Eq. (2).

\section{COMPARISON WITH MONTE CARLO SIMULATIONS}

We calculated the step-height distributions, propagation velocities, and spin-class populations, analytically and by kinetic MC simulations, for the phonon-assisted dynamic in the isotropic case, $J_{x}=J_{y}=J$. The details of our particular implementation of the $n$-fold way rejection-free $\mathrm{MC}$ algorithm ${ }^{28,29}$ are described in Refs. 5 and 7. The extension to continuous time, which is necessary to handle transition rates greater than unity, was introduced in Ref. 9.

The numerical results presented here are based on MC simulations mostly at the two temperatures, $T=0.2 T_{c}$ and $0.6 T_{c}\left[T_{c}=-2 J / \ln (\sqrt{2}-1) \approx 2.269 \mathrm{~J}\right.$ is the critical temperature for the isotropic, square-lattice Ising model $\left.{ }^{36}\right]$, with $L_{x}$ $=10000$ and fixed $\phi$ between 0 and $45^{\circ}$. (The tilt angle $\phi$ was fixed by imposing helical boundary conditions in the $x$ direction.) In order to ensure stationarity, we ran the simulation for $10^{4} n$-fold way updates per updatable spin (UPS) for thermalization before taking any measurements. Unless otherwise noted, the initial condition before thermalization was a microscopically flat interface. The initial condition only makes a difference near $H=0$. Stationary class populations and interface velocities were averaged over $10^{6}$ UPS. For the stronger fields at $T=0.2 T_{c}$, we used ten times as many UPS. Adequate statistics for the step-height pdfs were ensured by the large $L_{x}$.

\section{A. Stationary single-step probability densities}

Stationary single-step pdfs were obtained by MC simulation at $T=0.2 T_{c}$ and $0.6 T_{c}$ for $\phi=0$ and several values of $H$. The simulation data and the theoretical results for $p[\delta]$ are shown in Fig. 3. The theoretical results are calculated with Eq. (3), with $X(T, H)$ from Eq. (4). The agreement between theoretical and simulated results is quite good, particularly at the lower temperature.

Another way to compare the analytical and simulation results is by calculating $\langle|\delta|\rangle$ by averaging over the simulated step-height pdf and comparing these values with the theoretical ones obtained from Eq. (3), $\langle|\delta|\rangle=2 X /\left(1-X^{2}\right)$, with $X$ from Eq. (4). The results are shown in Fig. 4 for $\phi$ $=0$ at $T=0.2 T_{c}$ and $6.0 T_{c}$, calculated theoretically (solid lines) and by MC simulation (symbols). The agreement be- 

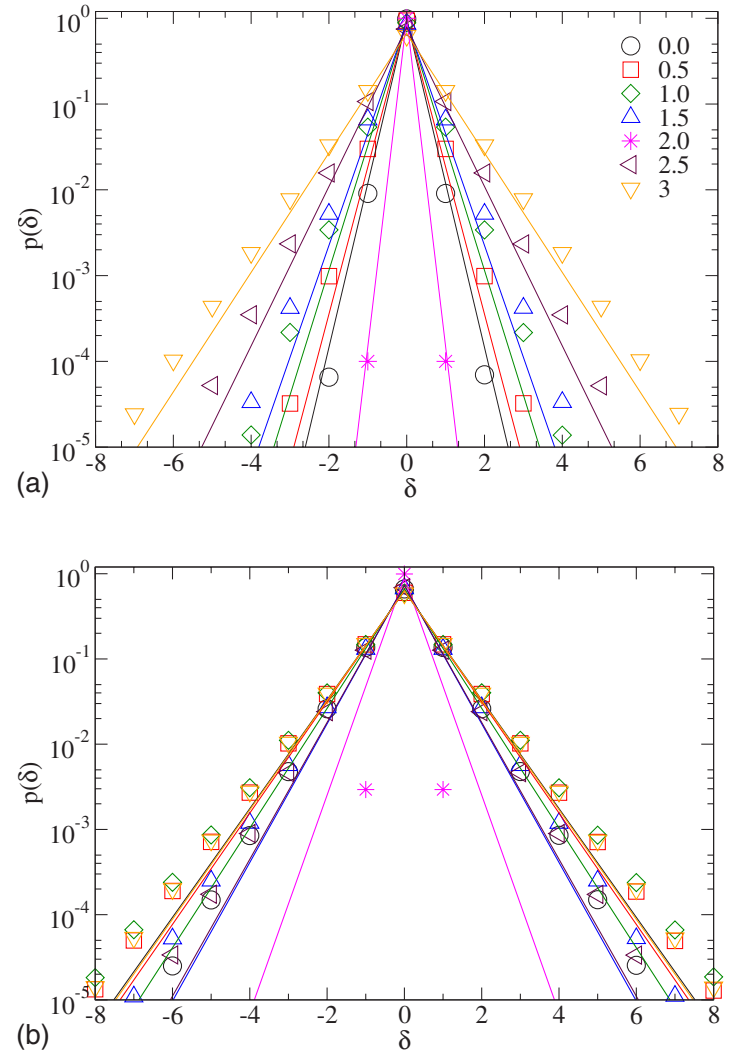

FIG. 3. (Color online) MC (data points) and analytical (solid lines) results for the stationary single-step pdf, shown on a logarithmic scale vs $\delta$, for $\phi=0$ and the values of $H / J$ given in the legend. (a) $T=0.2 T_{c}$ and (b) $T=0.6 T_{c}$. The symbols (and colors) have the same interpretations in (a) and (b). Note the nonmonotonic field dependence near $H / J=2$.

tween both results is reasonable. However, the theoretical data present a smoother dependence on the field than obtained from the simulation.

In Fig. 4, the behaviors of $\langle|\delta|\rangle$ near $H / J=0$ and 2 are of particular interest. At $H / J=0$, the system should be in equilibrium, and the theoretical results are exact. ${ }^{15}$ The discrepancy between theory and simulation at this field, especially at $T=0.6 T_{c}$, therefore means that the system simulated with the phonon-assisted dynamic, starting from a microscopically flat initial state, does not equilibrate completely. This is not due to a too short thermalization time. Rather, the reason is the aforementioned suppression by Eq. (2) near $H=0$ of transitions between states $11+$ and 11-, which correspond to diffusion of steps along the interface and represent an important mechanism for equilibration. However, when $\langle|\delta|\rangle$, near $H / J=0$ at $T=0.6 T_{c}$, is obtained by the phonon-assisted dynamic starting from the thermalized interface generated with the standard Glauber dynamic, there is excellent agreement with the theoretical result, as can also be seen in Fig. 4. As $H / J$ is increased from zero, the effect of the initial condition rapidly vanishes.

In Fig. 5(a), we show together snapshots of stationary interfaces at $H / J=0$ for $T=0.6 T_{c}$, generated in three different ways: using the standard Glauber dynamic, with the phononassisted dynamic starting from the equilibrated interface ob-

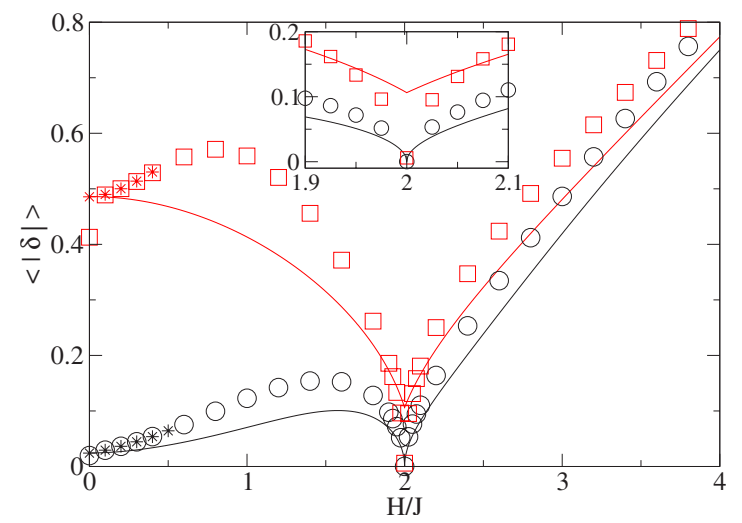

FIG. 4. (Color online) Average stationary step height $\langle|\delta|\rangle$ vs $H / J$ for $\phi=0$ at $T=0.2 T_{c}$ and $0.6 T_{c}$. The curves represent the theoretical results. Curve with circles (black): $T=0.2 T_{c}$. Curve with squares (gray, red online): $T=0.6 T_{c}$. These data refer to interfaces that started from a microscopically flat initial state. We also include values near $H / J=0$, calculated by the phonon-assisted dynamic, using as a starting state the thermalized interface obtained with the standard Glauber dynamic. Black asterisks: $T=0.2 T_{c}$. Gray asterisks (red online): $T=0.6 T_{c}$. The differences are evident only near $H / J$ $=0$. In this and all the following figures, the statistical uncertainty is much smaller than the symbol size. The inset shows a magnified view of the region around $H / J=2$. Note the disagreement between the theoretical and the simulation values at $T=0.6 T_{c}$ when $H / J=2$.

tained by the Glauber dynamic, and with the phonon-assisted dynamic starting from a microscopically flat interface. Due to the fact that energy-conserving moves (horizontal or vertical step diffusion) are prohibited by the phonon-assisted dynamic, the interface that started from the equilibrium interface is highly correlated with the latter, and both have $\langle|\delta|\rangle \approx 0.49$, the equilibrium value. For the same reason, the phonon-assisted interface that started from a microscopically flat interface configuration does not fully equilibrate but settles into a metastable configuration with $\langle|\delta|\rangle \approx 0.41$, as seen in Fig. 4.

At $H / J=2$, MC simulations give a value of $\langle|\delta|\rangle$ strictly zero for $T=0.2 T_{c}$ and very close to zero for $T=0.6 T_{c}$, while the theoretical value is very small but nonzero at $T=0.2 T_{c}$ and clearly larger at $T=0.6 T_{c}$ (see inset in Fig. 4). For strong values of the field, the step height is only weakly dependent on the temperature. These results are quite different from those obtained with the standard (hard) Glauber dynamic [see Fig. 5(a) of Ref. 7]. However, the strong $H$ dependence of the step heights is characteristic of hard dynamics.

In Fig. 5(b), we show together snapshots of stationary interfaces at $H / J=2$ and $T=0.6 T_{c}$, one thermalized with the standard Glauber dynamic, and the other generated by the phonon-assisted dynamic, using the Glauber interface as its starting state. The interface obtained with the phononassisted dynamic is almost entirely microscopically flat, with a very small density of "backward" (21-) notches that are created at a rate $\propto \exp (-8 J / T)$ and annihilated almost immediately. In fact, for $H / J=2$, the interface gets stuck as it can never progress beyond the absolute maximum of the starting configuration (and is thus nonergodic) due to the vanishing rate of the transition $01-\rightarrow 21+$. One possible way to over- 


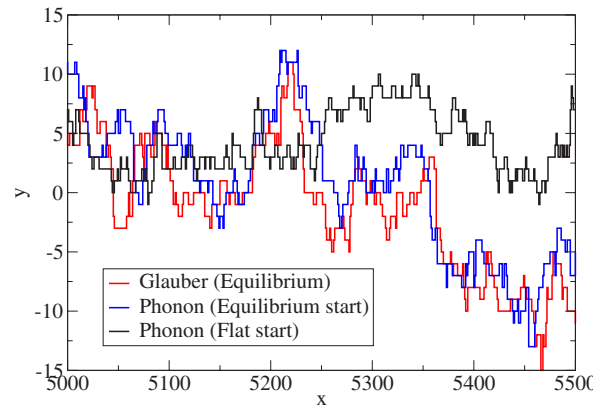

(a)

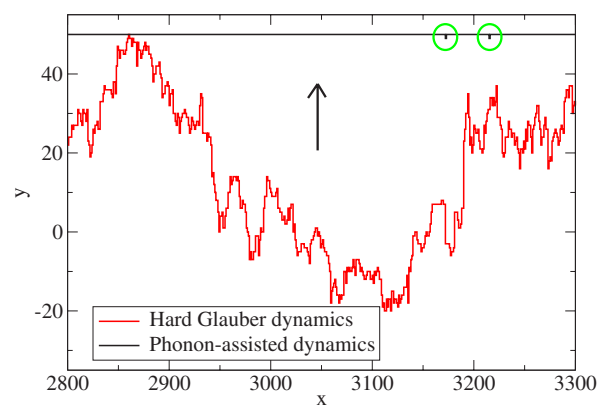

(b)

FIG. 5. (Color online) Short segments of thermalized interfaces for $\phi=0$. (a) $H / J=0, T=0.6 T_{c}$. The three graphs show an equilibrium interface created with the standard Glauber dynamic over $10^{4}$ UPS (medium gray, red online), an interface created by the phononassisted dynamic over $10^{6}$ UPS, using the equilibrium interface as a starting state (dark gray, blue online), and an interface created by the phonon-assisted dynamic over $10^{10}$ UPS, using a microscopically flat interface as starting state (black). (b) $H / J=2, T=0.6 T_{c}$. The jagged interface (medium gray, red online) is in the statistically stationary state, propagating in the direction of the arrow under the standard Glauber dynamic. At a given time, the dynamic is switched to the phonon-assisted transition rates, using the Glauber interface as initial state. The lagging parts rapidly catch up with the absolute maximum of the Glauber interface, where the interface gets permanently stuck in an almost perfect, microscopically flat configuration with a very small density of "backward" $12-$ notches (circled). At this field, the transition forbidden by the phonon-assisted dynamic is the nucleation of "forward" $12+$ notches, which are needed to nucleate propagation of a microscopically flat interface.

come this situation is to give the interface alternative paths to reach equilibrium. This could possibly be done by relaxing the SOS constraint to allow overhangs and bubbles. ${ }^{5,8}$ In contrast, the Glauber interface at the same field and temperature propagates at a nonzero velocity and is microscopically quite rough, with $\langle|\delta|\rangle \approx 1.79$.

\section{B. Stationary interface velocities}

In Fig. 6, we show the mean propagation velocity perpendicular to the interfaces vs $H / J$ for $\phi=0$, obtained with the analytical approximation, Eq. (8), and by simulations. In general, there is good agreement between the $\mathrm{MC}$ results and the nonlinear-response theory. However, there is a significant discrepancy at $H / J=2$. At this field, the simulated velocities are zero, independent of the temperature, while the theoretical value is small but nonzero and increases with tempera-

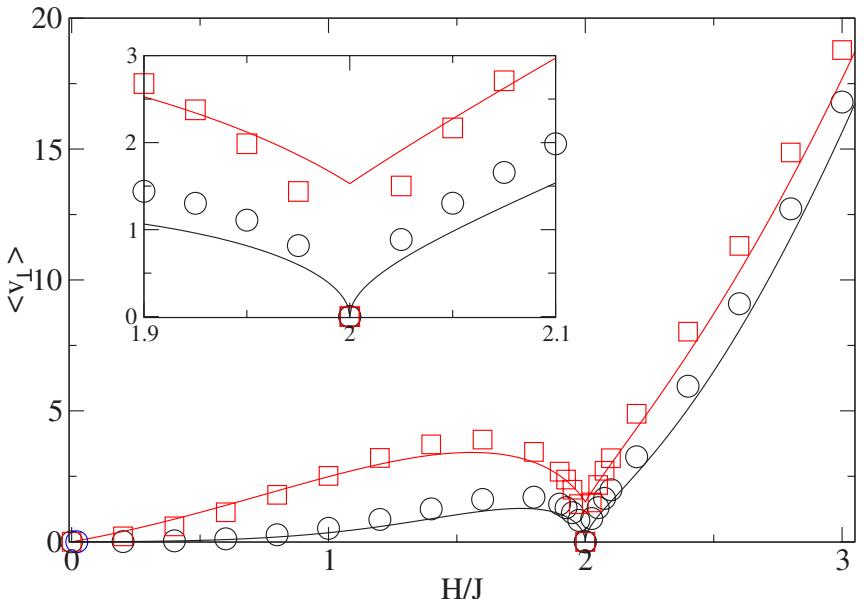

FIG. 6. (Color online) The average stationary normal interface velocity $\left\langle v_{\perp}\right\rangle$ vs $H / J$ for $\phi=0$. The MC results are shown as data points, circles for $T=0.2 T_{c}$ and squares for $T=0.6 T_{c}$, and the theoretical results as solid curves. The inset shows a magnified view of the region around $H / J=2$. Note again the disagreement between the theoretical and the simulation values at $T=0.6 T_{c}$ when $H / J=2$.

ture. This is perfectly consistent with the microscopically flat interface structure at $H / J=2$, discussed above. In fact, the knob-nucleating transition $01-\longrightarrow 21+$, which is forbidden at this field, is precisely the transition needed to nucleate the advance of a microscopically flat interface. When $H / J>2$, the velocity increases rather rapidly with $H$. This behavior is very different from the one obtained for other hard dynamics such as the standard Glauber and the TDA, where the velocity is bounded by unity [see Fig. 6 of Ref. 7 and Fig. 6(a) of Ref. 9].

In contrast to the results discussed above, Fig. 7 shows the velocity for the case in which the dimension of the phonon bath is unity, i.e., $d=1$, which has a transition rate that decreases smoothly and monotonically with $\Delta E / T$ (in contrast with the $d=2$ and 3 cases in which the transition rates vanish for $H=0$ and $H / J=2$ ). The agreement between theory and simulation is excellent over the whole range of $H / J$ and tilt angles, except for very large angles at higher fields. (This is also the case for step-height distributions and other characteristic quantities.)

The dependence of the normal velocity on the tilt angle $\phi$ is shown in Figs. 8(a) and 8(b) for several values of $H / J$ at $T=0.2 T_{c}$ and $T=0.6 T_{c}$, respectively. The agreement between the theoretical results and the simulations is very good except at higher fields, where the agreement is only good at intermediate values of $\phi$. The results are qualitatively similar to those obtained with other hard dynamics (see Refs. 7 and 9). At $T=0.2 T_{c}$, in weak fields the velocity increases with $\phi$, in agreement with the polynuclear growth model at small angles and the single-step model for larger angles. For strong fields, the behavior changes gradually to the reverse anisotropy of Eden-type models. ${ }^{37,38}$ This is essentially the same behavior observed for the TDA dynamic. ${ }^{9}$ At $T=0.6 T_{c}$, the velocity is nearly isotropic for weaker fields, while becoming Eden-like for stronger fields. The exception is the case $H / J$ $=2$, which at small angles presents a polynuclear growth type, as well as significant differences between the theoreti- 

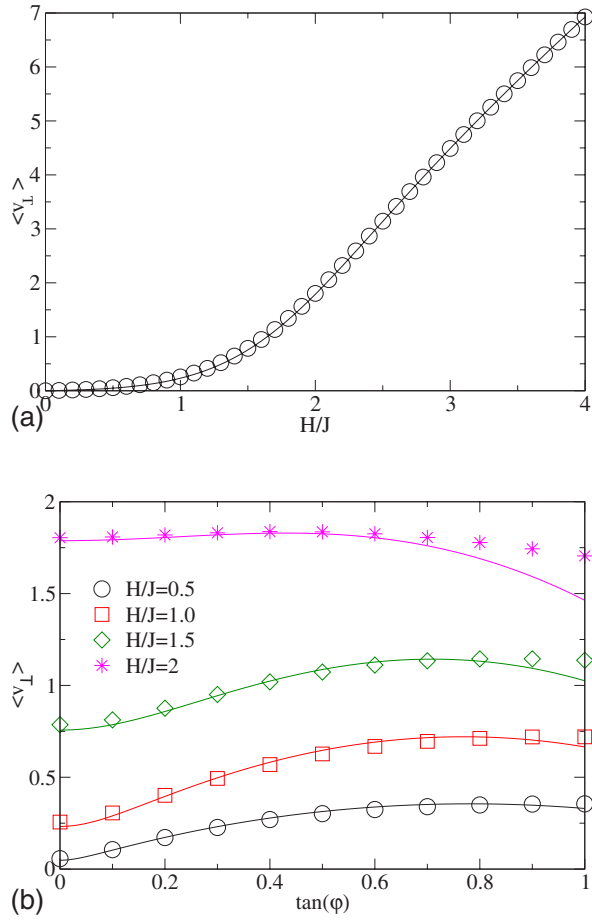

FIG. 7. (Color online) Average stationary normal interface velocity at $T=0.2 T_{c}$, obtained by coupling the system to a onedimensional phonon bath. MC data are represented by the symbols, and analytical results by the solid curves. (a) Velocity vs $H / J$ for $\phi=0$. (b) Velocity vs $\tan \phi$ for several values of $H / J$.

cal and simulated results. The behavior of the normal velocity at $T=0.6 T_{c}$ (excluding the case $H / J=2$ ) is very similar to that observed for both the TDA and the OSD dynamics. ${ }^{9}$

We have not performed simulations of cluster growth with this dynamic (except to some extent for nucleation at very low $T$ in Ref. 19). However, predictions for the asymptotic growth shapes could be obtained from the anisotropy curves for the growth velocity shown in Fig. 8 through a kinetic Wulff construction. ${ }^{39}$ While we shall not perform such a formal calculation here, we present the following qualitative predictions. Isotropy should lead to near-circular growth shapes, while anisotropic growth velocities should lead to the elimination of the faster-growing faces. As a result, the "normal" anisotropy with growth velocity increasing with $\phi$ should lead to approximately square growth shapes with faces parallel to the coordinate axes. At $H / J=2$, the results shown in Fig. 5(b) lead us to expect any initial cluster to evolve to a stationary rectangle bounded by planes touching the initial cluster at its extrema in the $x$ and $y$ directions. In contrast, Eden-like "reverse" anisotropy should lead to approximately diamond-shaped clusters with faces making $45^{\circ}$ angles with the axes.

The temperature dependence of the normal interface velocity is shown in Fig. 9 for several values of $H / J$. The agreement between the simulations and the analytical results is reasonable, except for $H / J=2$, where the simulated velocity remains zero for all temperatures, while the predicted velocity increases monotonically with the temperature. This discrepancy is also due to the static and microscopically flat interface that forms at $H / J=2$. This figure also shows that as
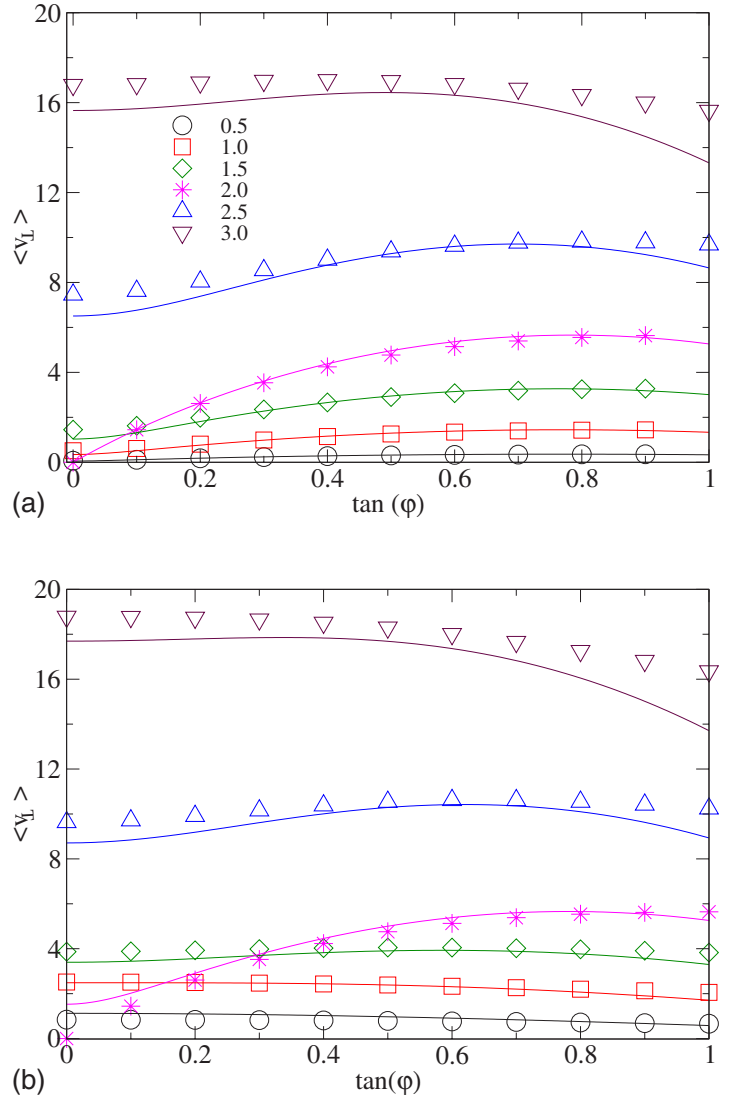

FIG. 8. (Color online) The average stationary normal interface velocity $\left\langle v_{\perp}\right\rangle$ vs $\tan \phi$, for several values of $H / J$. The symbols represent MC data, and the solid curves analytical results. (a) $T$ $=0.2 T_{c}$ and (b) $T=0.6 T_{c}$. The symbols have the same interpretations in (a) and (b), given by the legend in (a). Online, the colors of the curves and symbols match.

$T \rightarrow 0$, the system develops a step discontinuity: the velocity is zero for $H / J \leqslant 2$ and increases with $H$ for stronger fields. This discontinuity at $T=0$ is also observed with the TDA $^{9}$ and with the standard Glauber dynamic (see Fig. 8 of Ref. 7).

\section{Spin-class populations and skewness}

To test the analytical assumption that different steps are statistically independent, we compare the analytical results for the mean class populations ${ }^{7}$ with the simulated ones. The six mean class populations, $\langle n(01 s)\rangle,\langle n(11 s)\rangle$, and $\langle n(21 s)\rangle$ with $s= \pm 1$, for $\phi=0$ at $T=0.2 T_{c}$ and $T=0.6 T_{c}$ are shown vs $H$ in Fig. 10. At both temperatures, the analytical approximations follow the average of the populations for $s=+1$ and $s=-1$ qualitatively well. However, for small fields, $H / J<2$, the simulations show a stronger dependence on $H$ than the mean-field results. This difference is more evident at the higher temperature, where the simulations show that the population in front of the interface $(s=-1)$ is quite different from the one behind it $(s=+1)$. Well away from the special fields $(H / J=0$ and 2$)$, the interfaces are a little rougher than the theory predicts (lower $01 s$ and higher $11 s$ populations). Near the special fields, the interfaces appear to get caught in smoother (metastable for $H / J=0$ ) configurations. For $H / J$ $=0$, the $01 \mathrm{~s}$ populations are slightly higher than the theoreti- 


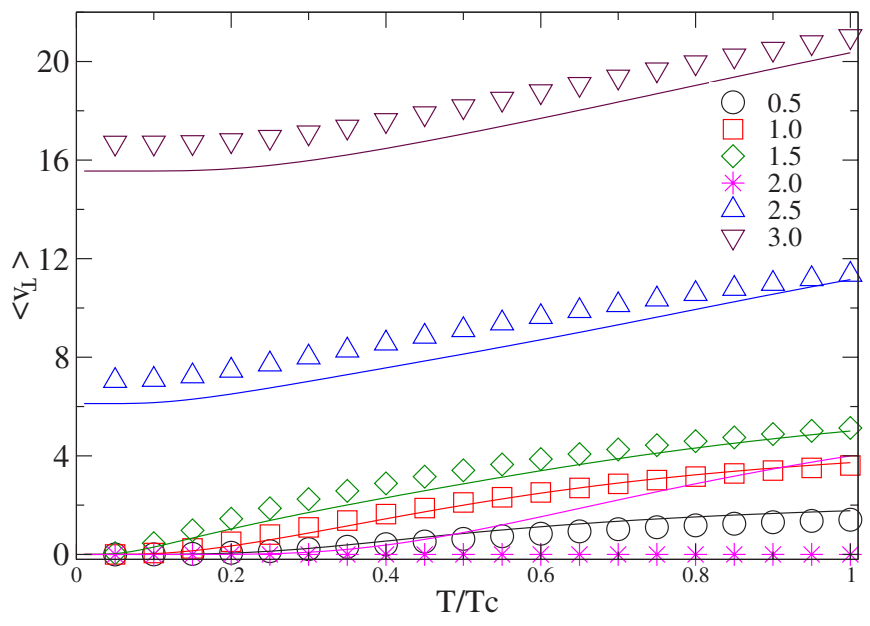

FIG. 9. (Color online) The average stationary normal interface velocity $\left\langle v_{\perp}\right\rangle$ vs $T$ for $\phi=0$ and $H / J$ between 0.5 and 3 . MC data are represented by data points and analytical results by solid curves. Online, the colors of the curves and symbols match. The agreement between simulation and theory is quite good, except for $H / J=2$.

cally predicted value, and the $11 \mathrm{~s}$ populations are slightly lower. This is more evident at the higher temperature. Note that when $H / J=0$, the interface should be in equilibrium, and the theoretical value is exact. However, this exact value is only reached if the interface is equilibrating properly. This can be seen in Fig. 10(b), where we include the population averages, both calculated starting from a microscopically flat interface and from a thermalized interface generated with the standard Glauber dynamic. For $H / J=2$, the interface is much smoother than predicted. [For $T=0.6 T_{c}$, the measured values for $\langle n(01 \pm)\rangle,\langle n(11-)\rangle$, and $\langle n(11+)\rangle$ are approximately $0.9999,10^{-4}$, and 0 , compared with the respective predicted values of $0.9,0.1$, and 0.01 .]

The short-range correlations between neighboring steps are responsible for the skewness between the spin populations on the leading and trailing edges of the interface that appears in the simulation results. This phenomenon is commonly observed in driven interfaces. It occurs even when the long-range correlations vanish as they do for interfaces in the KPZ dynamic universality class, to which the present model belongs for all finite, nonzero values of $H / J \neq 2$. Skewness has also been observed in several other SOS-type models, such as the body-centered SOS model studied by Neergaard and den Nijs, ${ }^{40}$ the model for step propagation on crystal surfaces with a kink-Ehrlich-Schwoebel barrier studied by Pierre-Louis et al., ${ }^{41}$ and a model for the local time horizon in parallel kinetic MC simulations studied by Korniss et al. ${ }^{42}$ No skewness was observed for the SOS model with the soft Glauber dynamic. ${ }^{6}$ However, some skewness was present in the OSD model, ${ }^{9}$ indicating that a complete lack of skewness is not a necessary characteristic of soft dynamics. Also, a small degree of skewness was observed for the Ising model (whose interfaces include bubbles and overhangs) with the soft Glauber dynamic (about 2 orders of magnitude smaller than the skewness observed for the hard Glauber dynamic). ${ }^{8}$ The TDA dynamic also presents considerable skewness. ${ }^{9}$ The correlations associated with the skew-
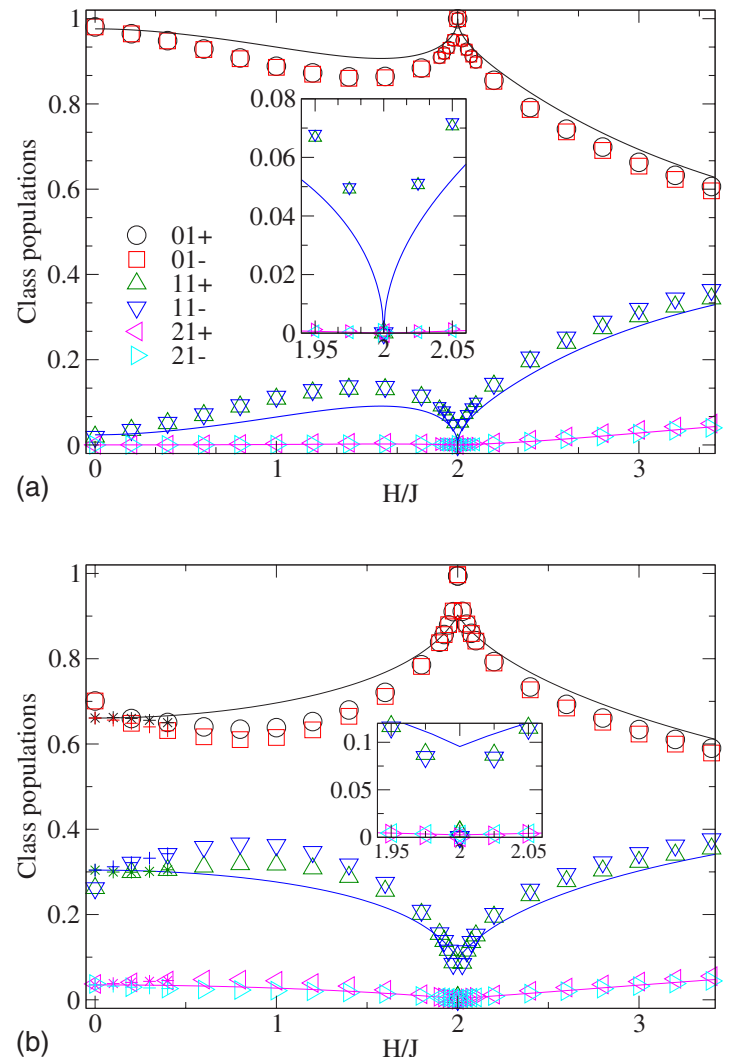

FIG. 10. (Color online) Mean stationary class populations $\langle n(j k s)\rangle$ vs $H / J$ for $\phi=0$. The simulation results are indicated by symbols, and the analytic approximations by solid curves. (a) $T=0.2 T_{c}$ and (b) $T=0.6 T_{c}$. At $T=0.6 T_{c}$, we also include some values [asterisks for $\langle n(j k+)\rangle$ and pluses for $\langle n(j k-)\rangle]$ calculated with the interface created by the phonon-assisted dynamic, using the thermalized interface obtained by the Glauber dynamic as starting state. Note that in this case, there is excellent agreement between theory and simulations at $H / J=0$. The other symbols have the same interpretations in (a) and (b), given by the legend in (a). The insets in both (a) and (b) show $\langle n(11 s)\rangle$ and $\langle n(21 s)\rangle$ near $H / J=2$.

ness generally lead to a broadening of protrusions on the leading edge ("hilltops"), while those on the trailing edge ("valley bottoms") are sharpened 40 or the other way around. ${ }^{42}$ In terms of spin-class populations, the former corresponds to $\langle n(21-)\rangle\rangle\langle n(21+)\rangle$ and $\langle n(11+)\rangle\rangle\langle n(11-)\rangle$. The relative skewness can therefore be quantified by the two functions, ${ }^{40}$

$$
\rho=\frac{\langle n(21-)\rangle-\langle n(21+)\rangle}{\langle n(21-)\rangle+\langle n(21+)\rangle}
$$

and $^{7}$

$$
\epsilon=\frac{\langle n(11+)\rangle-\langle n(11-)\rangle}{\langle n(11+)\rangle+\langle n(11-)\rangle} .
$$

These two skewness parameters are shown together in Fig. 11. The temperature dependence of the skewness is stronger at the lower temperature and smaller fields, and it is especially pronounced for $\rho$, due to the low concentration of sites in the class $21+$ at low temperatures and weak fields. $\epsilon$ is 


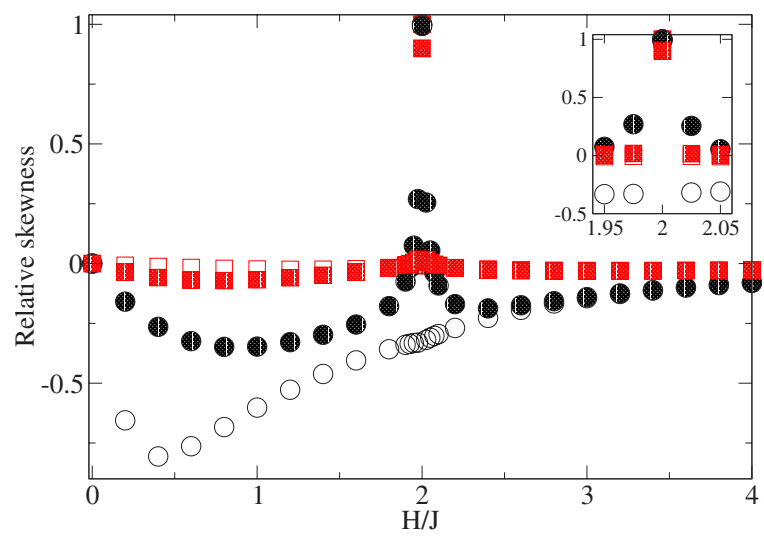

FIG. 11. (Color online) The two relative skewness parameters $\rho$ (circles, black) and $\epsilon$ (squares, red online), defined in Eqs. (9) and (10), respectively. The parameters are shown vs $H / J$ for $\phi=0$, at $T=0.2 T_{c}$ (empty symbols) and at $T=0.6 T_{c}$ (filled symbols). The inset shows a magnified view of the region around $H / J=2$.

very small and almost independent of $T$ and $H$, except at $H / J=2.0$, where both values are near unity, consistent with the picture of an interface with a very low density of 21notches.

\section{DISCUSSION AND CONCLUSIONS}

In this paper, we have studied the nanostructure of an unrestricted SOS interface interacting with a twodimensional phonon heat bath and driven far from equilibrium by an applied field. This work is a continuation of previous studies aimed to explore the crucial role of the stochastic dynamics selected to simulate physical systems. ${ }^{5-9,43,44}$ Important properties such as nanostructure and mobility of driven interfaces have been shown to be strongly dependent on the stochastic dynamics. ${ }^{5-9}$

The transition rates that give the evolution of the system are calculated by coupling the spin Hamiltonian to a twodimensional phonon heat bath. The dynamic generated in this way belongs to the class known as hard. ${ }^{10}$ In condensedmatter systems, phonon-driven dynamics are dominant, and technologically they are becoming increasingly important, e.g., in connection with the behavior of quantum dots. ${ }^{4}$

We studied the nanostructure and velocity of the SOS interface by kinetic MC simulations and by a nonlinear mean-field theory developed in previous papers. 5,7 We calculated the interface velocity as a function of the driving field, temperature, and angle of the interface relative to the lattice axes. We also studied the local shape of the interface in terms of the spin-class populations, the average height of a step, and the probability density for individual steps in the interface.

In general, we found good agreement between the theoretical calculations and the MC simulations. In particular, we found the strong dependence of the interface structure on the field characteristic of systems that evolve under hard dynamics, such as the Glauber or TDA dynamics. ${ }^{5,7,9}$

Our theoretical results are based on the mean-field assumption that individual steps of the interface are statisti- cally independent; short-range correlations are neglected. However, our MC results show asymmetry between the spin populations on the leading and trailing edges of the interface, which is an indication of the existence of such short-range correlations. With increasing field, the interfaces undergo a gradual breakdown of up-down symmetry, which has also been observed in other examples of driven interfaces. ${ }^{9,40-42}$ Aside from such, relatively minor, discrepancies between the theoretical mean-field predictions and the simulation results, which show that there is room for improvement of the meanfield model, the theory predicts very accurately the qualitative behavior of the interfaces and yields a reasonable overall approximation to their quantitative behavior. The important exceptions are the special field values, $H / J=0$ and 2 , where certain transitions allowed by the SOS constraint are forbidden by the phonon-assisted dynamic for phonon baths of dimension greater than 1 . At $H / J=0$, this leads to a failure of the simulated interface to reach thermal equilibrium, while at $H / J=2$, it leads to an abnormally flat interface that is unable to propagate.

It should, however, be noted that the phonon-assisted dynamic defined by Eq. (2) is based on a weak, linear coupling of the bosonic bath to the spins. It is therefore possible that higher-order and/or multiphonon corrections to the transition rates could restore the vanishing rates for energy-conserving transitions. Nevertheless, higher-order effects may not completely mask the slowing down of the interface in the vicinity of the special field values observed here. We therefore expect that much of the characteristic field dependence will carry over to more sophisticated rate models. This expectation is supported by the results of recent experiments on phononmediated spin dynamics in a quantum dot, ${ }^{4}$ in which significant slowing down was observed for nearly energyconserving transitions. Another interesting question is to what extent a relaxation of the SOS constraint to consider a full Ising interface including overhangs and bubbles (see Refs. 5 and 8) might open alternative channels for full equilibration. Naturally, one can also think of more general dynamics, including multiple-spin-flip elementary transitions. These questions are left for future study.

As in previous studies, ${ }^{5-9,43,44}$ our results indicate strong differences between interfaces moving under different stochastic dynamics, emphasizing the need for extreme care in selecting the appropriate dynamic for the physical system of interest. This general understanding and the specific results for the phonon-assisted dynamic presented in this paper represent significant steps in the direction of putting kinetic MC simulations on a solid physical foundation.

\section{ACKNOWLEDGMENTS}

G.M.B. appreciates the hospitality of the School of Computational Science at Florida State University. This research was supported in part by National Science Foundation Grants Nos. DMR-0240078 and DMR-0444051, by Florida State University through the Center for Materials Research and Technology and the School of Computational Science, by the National High Magnetic Field Laboratory, and by the Deanship of Research and Development of Universidad Simón Bolívar. 
*buendia@usb.ve

†rikvold@scs.fsu.edu

kolesik@acms.arizona.edu

§kyungwha@pontiac.phys.vt.edu

"man40@ra.msstate.edu

${ }^{1}$ A.-L. Barabási and H. E. Stanley, Fractal Concepts in Surface Growth (Cambridge University Press, Cambridge, 1995), detailed discussion and copious references to the theoretical and experimental literature can be found here.

${ }^{2}$ P. Meakin, Fractals, Scaling, and Growth Far From Equilibrium (Cambridge University Press, Cambridge, 1998) and references therein.

${ }^{3}$ M. Thorwart, J. Eckel, and E. R. Mucciolo, Phys. Rev. B 72, 235320 (2005).

${ }^{4}$ T. Meunier, I. T. Vink, L. H. Willems, K.-J. Tielrooij, R. Hanson, F. H. L. Koppens, H. P. Tranitz, W. Wegscheider, L. P. Kouwenhoven, and L. M. K. Vandersypen, Phys. Rev. Lett. 98, 126601 (2007).

${ }^{5}$ P. A. Rikvold and M. Kolesik, J. Stat. Phys. 100, 377 (2000).

${ }^{6}$ P. A. Rikvold and M. Kolesik, J. Phys. A 35, L117 (2002).

${ }^{7}$ P. A. Rikvold and M. Kolesik, Phys. Rev. E 66, 066116 (2002).

${ }^{8}$ P. A. Rikvold and M. Kolesik, Phys. Rev. E 67, 066113 (2003).

${ }^{9}$ G. M. Buendía, P. A. Rikvold, and M. Kolesik, Phys. Rev. B 73, 045437 (2006); J. Mol. Struct.: THEOCHEM 769, 189 (2006).

${ }^{10}$ J. Marro and R. Dickman, Nonequilibrium Phase Transitions in Lattice Models (Cambridge University Press, Cambridge, 1999).

${ }^{11}$ T. Ala-Nissila and S. C. Ying, Prog. Surf. Sci. 39, 227 (1992).

${ }^{12}$ T. Ala-Nissila, J. Kjoll, and S. C. Ying, Phys. Rev. B 46, 846 (1992).

${ }^{13}$ H. C. Kang and W. H. Weinberg, J. Chem. Phys. 90, 2824 (1989).

${ }^{14}$ K. A. Fichthorn and W. Weinberg, J. Chem. Phys. 95, 1090 (1991).

${ }^{15}$ W. K. Burton, N. Cabrera, and F. C. Frank, Philos. Trans. R. Soc. London, Ser. A 243, 299 (1951).

${ }^{16}$ M. Kardar, G. Parisi, and Y.-C. Zhang, Phys. Rev. Lett. 56, 889 (1986).

${ }^{17}$ K. Saito, S. Takesue, and S. Miyashita, Phys. Rev. E 61, 2397 (2000).

${ }^{18}$ K. Park and M. A. Novotny, Comput. Phys. Commun. 147, 737
(2002).

${ }^{19}$ K. Park, M. A. Novotny, and P. A. Rikvold, Phys. Rev. E 66, 056101 (2002).

${ }^{20}$ M. J. Henrich, M. Michel, M. Hartmann, G. Mahler, and J. Gemmer, Phys. Rev. E 72, 026104 (2005).

${ }^{21}$ K. Park (unpublished).

${ }^{22}$ L. Solomon, M. A. Novotny, S. Caliskan, K. Park, and P. A. Rikvold (unpublished).

${ }^{23}$ P. A. Martin, J. Stat. Phys. 16, 149 (1977).

${ }^{24}$ V. M. Axt, K. Victor, and A. Stahl, Phys. Rev. B 53, 7244 (1996).

${ }^{25}$ C. N. Yang and T. D. Lee, Phys. Rev. 87, 404 (1952).

${ }^{26}$ R. K. Pathria, Statistical Mechanics, 2nd ed. (ButterworthHeinemann, Oxford, 1996).

${ }^{27}$ H. Spohn, J. Stat. Phys. 71, 1081 (1993).

${ }^{28}$ G. H. Gilmer, J. Cryst. Growth 35, 15 (1976).

${ }^{29}$ A. B. Bortz, M. H. Kalos, and J. L. Lebowitz, J. Comput. Phys. 17, 10 (1975).

${ }^{30}$ M. A. Novotny, Comput. Phys. 9, 46 (1995).

${ }^{31}$ P. Devillard and H. Spohn, Europhys. Lett. 17, 113 (1992).

${ }^{32}$ P. Meakin, P. Ramanlal, L. M. Sander, and R. C. Ball, Phys. Rev. A 34, 5091 (1986).

${ }^{33}$ M. Plischke, Z. Rácz, and D. Liu, Phys. Rev. B 35, 3485 (1987).

${ }^{34}$ J. Krug and H. Spohn, Europhys. Lett. 8, 219 (1989).

${ }^{35}$ J. Kertész and D. E. Wolf, Phys. Rev. Lett. 62, 2571 (1989).

${ }^{36}$ L. Onsager, Phys. Rev. 65, 117 (1944).

${ }^{37}$ P. Meakin, R. Jullien, and R. Botet, Europhys. Lett. 1, 609 (1986).

${ }^{38}$ R. Hirsch and D. E. Wolf, J. Phys. A 19, L251 (1986).

${ }^{39}$ D. Du and D. J. Srolovitz, J. Cryst. Growth 296, 86 (2006) and references therein.

${ }^{40}$ J. Neergaard and M. den Nijs, J. Phys. A 30, 1935 (1997).

${ }^{41}$ O. Pierre-Louis, M. R. D’Orsogna, and T. L. Einstein, Phys. Rev. Lett. 82, 3661 (1999).

${ }^{42}$ G. Korniss, Z. Toroczkai, M. A. Novotny, and P. A. Rikvold, Phys. Rev. Lett. 84, 1351 (2000).

${ }^{43}$ K. Park, P. A. Rikvold, G. M. Buendía, and M. A. Novotny, Phys. Rev. Lett. 92, 015701 (2004).

${ }^{44}$ G. M. Buendía, P. A. Rikvold, K. Park, and M. A. Novotny, J. Chem. Phys. 121, 4193 (2004). 